\title{
Deleterious mutation in FDX1L gene is associated with a novel mitochondrial muscle myopathy
}

\author{
Ronen Spiegel ${ }^{\star, 1,2,5}$, Ann Saada ${ }^{3,5}$, Jonatan Halvardson ${ }^{4}$, Devorah Soiferman ${ }^{3}$, Avraham Shaag ${ }^{3}$, \\ Simon Edvardson ${ }^{3}$, Yoseph Horovitz ${ }^{1}$, Morad Khayat ${ }^{2}$, Stavit A Shalev ${ }^{2}$, Lars Feuk ${ }^{4}$ and Orly Elpeleg ${ }^{3}$
}

Isolated metabolic myopathies encompass a heterogeneous group of disorders, with mitochondrial myopathies being a subgroup, with depleted skeletal muscle energy production manifesting either by recurrent episodes of myoglobinuria or progressive muscle weakness. In this study, we investigated the genetic cause of a patient from a consanguineous family who presented with adolescent onset autosomal recessive mitochondrial myopathy. Analysis of enzyme activities of the five respiratory chain complexes in our patients' skeletal muscle showed severely impaired activities of iron sulfur (Fe-S)-dependent complexes I, II and III and mitochondrial aconitase. We employed exome sequencing combined with homozygosity mapping to identify a homozygous mutation, c.1A $>$ T, in the FDX1L gene, which encodes the mitochondrial ferredoxin 2 (Fdx2) protein. The mutation disrupts the ATG initiation translation site resulting in severe reduction of Fdx2 content in the patient muscle and fibroblasts mitochondria. Fdx2 is the second component of the Fe-S cluster biogenesis machinery, the first being IscU that is associated with isolated mitochondrial myopathy. We suggest adding genetic analysis of FDX1L in cases of mitochondrial myopathy especially when associated with reduced activity of the respiratory chain complexes I, II and III. European Journal of Human Genetics (2014) 22, 902-906; doi:10.1038/ejhg.2013.269; published online 27 November 2013

Keywords: FDX1L gene; Fe-S cluster; respiratory chain; metabolic myopathy

\section{INTRODUCTION}

Metabolic myopathies comprise a heterogeneous group of inherited disorders that resulting in skeletal muscle dysfunction because of insufficient energy production. The most common clinical presentation encountered is acute, often recurrent, exercise-induced episodes of myalgia and muscle cramps often culminating in rhabdomyolysis and myoglobinuria. Alternatively, they may manifest with fixed, often progressive, muscle weakness. Metabolic myopathies may be grossly divided into three main etiological groups. The two major groups are defects in muscle glycogen breakdown (muscle glycogenoses) and beta oxidation disorders, both disrupt the two principal skeletal muscle energy sources, that is, glycogen and free fatty acids. The third group consists of mitochondrial myopathies involving intra-mitochondrial ATP production via the five respiratory chain complexes.

Herein, we report the results of the molecular investigation of a patient who presented with recurrent myoglobinuria and slowly progressive muscle weakness.

\section{MATERIALS AND METHODS \\ Subjects \\ The affected individual was a 15-year-old female, the fourth child of first degree cousins of Jewish Moroccan origin (Figure 1a). The patient and her five family members were enrolled in this study. All participants signed a written informed consent form before enrollment (minors were signed by their parents after detailed explanations). The study was approved by the local institutional review boards.}

\section{Biochemical assay of OXPHOS}

Enzymatic activities of the five respiratory chain complexes, rotenone-sensitive $\mathrm{NADH} \mathrm{CoQ}$ reductase (complex I), succinate cytochrome $c$ reductase (complex II + III), succinate dehydrogenase (SDH; complex II), cytochrome $c$ oxidase (complex IV) and $\mathrm{Mg}^{+}{ }^{+}$ATPase (complex V) were determined in isolated muscle mitochondria and fibroblast homogenate from the patient as we have previous described. ${ }^{1}$ Citrate synthase, a mitochondrial matrix enzyme marker and mitochondrial aconitase and lipoamide dehydrogenase were measured by spectrophotometric assays. ${ }^{2}$ All spectrophotometric measurements were carried out using a double beam spectrophotometer (UVIKON 930, Secomam, France). Pyruvate dehydrogenase complex (PDHc) activity in muscle homogenate was measured using ${ }^{14} \mathrm{C}$-labeled pyruvate as substrate. ${ }^{2}$

\section{Exome sequencing}

Exome capture was performed using the Agilent SureSelect All Exon $50 \mathrm{Mb}$ kit (Agilent Technologies, Santa Clara, CA, USA) according to the manufacturer's protocol. Sequencing was performed using $1 / 2$ lane on a SOLiD5500XL instrument (Life Technologies, Carlsbad, CA, USA). The analysis suite LifeScope 2.5 (Applied Biosystems, Foster City, CA, USA) was used to align sequence reads to the human reference assembly GRCh37/hg19 and to call single-nucleotide variants (SNVs) and small insertion/deletions (Indels). Called variants were further annotated using $\mathrm{ANNOVAR}^{3}$ and filtered against dbSNP135. ${ }^{4}$ Non-synonymous variants were analyzed using MutationTaster ${ }^{5}$ and PolyPhen ${ }^{6}$ to assign scores to each variant to predict the effect of the variant on protein functionality. The pathogenic variant identified was submitted to the $\mathrm{LOVD}^{3}$ database (http://databases. lovd.nl/shared/genes).

${ }^{1}$ Department of Pediatric A', Emek Medical Center, Afula, Rappaport School of Medicine, Technion, Haifa, Israel; ${ }^{2}$ Genetic Institute, Emek Medical Center, Afula, Rappaport School of Medicine, Technion, Haifa, Israel; ${ }^{3}$ Monique and Jacques Roboh Department of Genetic Research, Hadassah, Hebrew University Medical Center, Jerusalem, Israel; ${ }^{4}$ Department of Immunology, Genetics and Pathology, Science for Life Laboratory Uppsala, Rudbeck Laboratory, Uppsala University, Uppsala, Sweden

*Correspondence: Dr R Spiegel, Department of Pediatrics A, Emek Medical Center, Afula, Rappaport School of Medicine, Technion, Afula 18101, Israel. Tel: +972-4-6494216; Fax:+972-4-6495251; E-mails: spiegelr@zahav.net.il or spiegel_ro@clalit.org.il

${ }^{5}$ These authors contributed equally to this work.

Received 23 March 2013; revised 4 October 2013; accepted 25 October 2013; published online 27 November 2013 
a

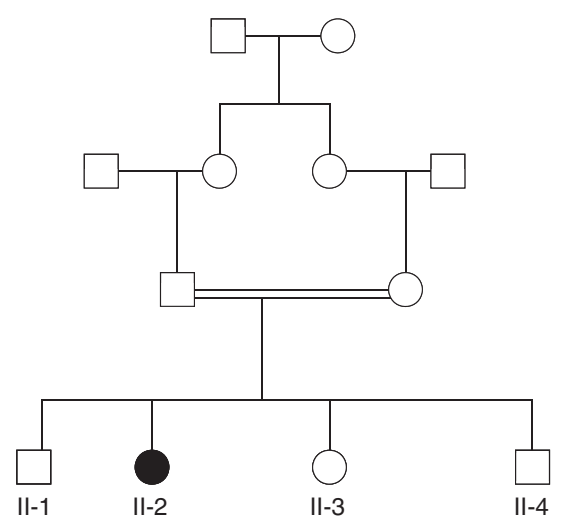

Affected b

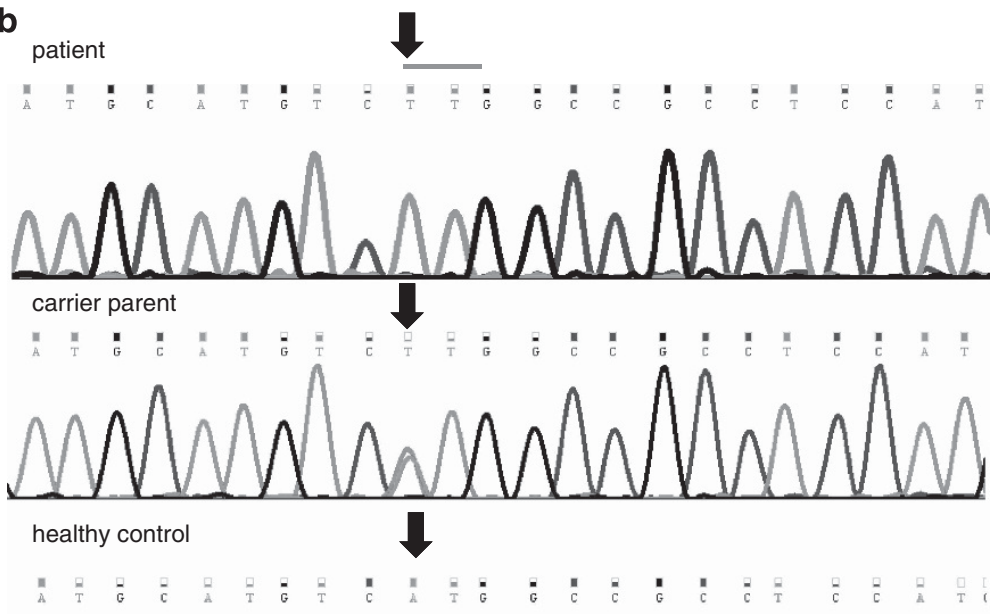

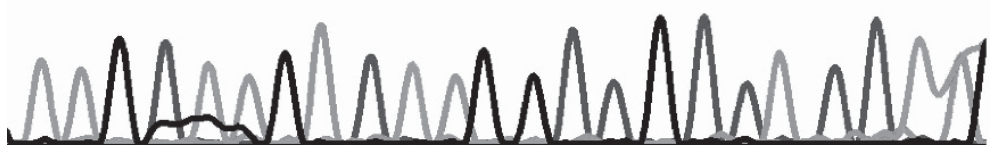

Figure 1 (a) Pedigree of family. Affected individual is represented by filled figure. (b) DNA sequence showing in the lower case the wild-type sequence and in the upper case substitution of A to T (arrow) disrupting initial Met formation. Carrier parent is represented in the middle panel.

\section{Immunobloting}

Muscle and fibroblast mitochondria were separated by SDS-polyacrylamide gel electrophoresis on a $14 \%$ gel, transferred to nitrocellulose membranes and probed with mouse-anti-SDHA, mouse-anti-COX II antibodies (Molecular Probes, Eugene, OR, USA) or rabbit-anti- ferredoxin 2 (Fdx2; a generous gift from Prof Roland Lill, Marburg) with secondary horseradish peroxidaseconjugated antibodies (Jackson Immunoresearch Laboratories, West Grove, PA, USA) using enhanced chemiluminescence according to the manufacturer's instruction (EZ-ECL kit, Biological Industries, Beit Haemek, Israel). Band intensities were analyzed using the Image J software (http://imagej.nih.gov/ij/).

\section{RESULTS}

\section{Clinical report}

The patient was born following uneventful pregnancy and delivery. She began to walk independently at 13 months of age and developed normally since then. Currently, she attends regular school. She was normally active and had normal exercise tolerance until the age of 12 years when she experienced her first episode of severe proximal lower limb weakness accompanied by muscle cramps and myoglobinuria. On admission, she was severely fatigued had clear Gower sign and waddling gait with inability to climb stairs unaided. In addition, she developed shortness of breath while walking for more than $20 \mathrm{~m}$. Laboratory studies revealed serum creatine kinase (CK) of $31000 \mathrm{IU} / 1$ (normal range $<200 \mathrm{IU} / \mathrm{l}$ ), lactic acidosis with serum lactate of $7.8 \mathrm{mmol} / \mathrm{l}$ (normal range $<2.0 \mathrm{mmol} / \mathrm{l}$ ), but normal arterial blood gases. Urinary organic acid assay disclosed extensive excretion of lactate, 3-methyl glutaconic acid $(178 \mu \mathrm{mol} / \mathrm{mmol}$ creat, control $<20$ $\mu \mathrm{mol} / \mathrm{mmol}$ creat), ketones and Krebs cycle metabolites. Renal functions including serum urea and creatinine were normal. Treatment was started with intravenous fluids and bicarbonate resulting in gradual decrease of CK levels and resolution of myoglobinuria. Accordingly, proximal muscle weakness improved allowing steady gait during her 6th day of admission. On discharge, she had no fatigue, negative Gower sign and she was able to climb two flights of stairs without rest or shortness of breath.

Despite a seemingly complete clinical recovery accompanied by normalization of serum CK, lactate levels remained elevated ranging between 4 and $6 \mathrm{mmol} / \mathrm{l}$ and urinary 3-methyl glutaconic acid remained mildly elevated ranging between 20 and $40 \mu \mathrm{mol} / \mathrm{mmol}$ creat. A year after her first admission, she developed a second episode of myoglobinuria and severe muscle weakness induced by 1 month of myalgia and muscle cramps, which appeared after regular daily activities. This time recovery lasted longer and the patient remained with mild degree of lower limb girdle weakness as well as increased serum CK fluctuating between 400 and $3000 \mathrm{IU} / \mathrm{l}$, and constantly elevated serum lactate. On her last physical examination, the patient displayed steady gait, no Gower sign, normally elicited tendon reflexes, and normal muscle mass without evidence of pseudohypertrophy. She continues regular activities but avoids sport classes at school. Although climbing she needs to rest after two flights of stairs and she becomes fatigued after walking approximately $500 \mathrm{~m}$ consistent with a slowly progressive course of proximal myopathy. A therapeutic trial with coenzyme Q10, riboflavin and thiamin for several months did not result in any clinical improvement.

Laboratory investigation included complete blood count, glucose, renal functions, lipid profile, ammonia, thyroid functions, carnitine and acylcarnitines, amino acids, very-long-chain fatty acids and transferrin isoelectric focusing all of which were normal. Plasma concentration of fibroblast growth factor 21 was borderline elevated to $338 \mathrm{pg} / \mathrm{ml}$ (control $250 \mathrm{pg} / \mathrm{ml}$ ) consistent with mitochondrial impairment. $^{7}$ Enzymatic activity of alpha glucosidase in lymphocytes was normal. Molecular analysis of the coding exons of ISCU gene and PDHA1 gene disclosed normal sequence. Annual cardiologic and echocardiographic examination since her first admission remained normal.

\section{Biochemical assay of muscle OXPHOS}

Muscle biopsy performed in the patient while she was 3 months after complete recovery, disclosed normal histology by light and electron microscopy. The enzymatic activities of the iron sulfur (Fe-S)dependent respiratory chain complexes I, II, II + III and III in isolated mitochondria were markedly decreased to $6-36 \%$ of normal, with complex II being the most affected. In contrast, complexes IV and V showed increased activities (Figure 2a). The activity of aconitase, a matrix Fe-S enzyme, was also reduced to $25 \%$, whereas citrate 


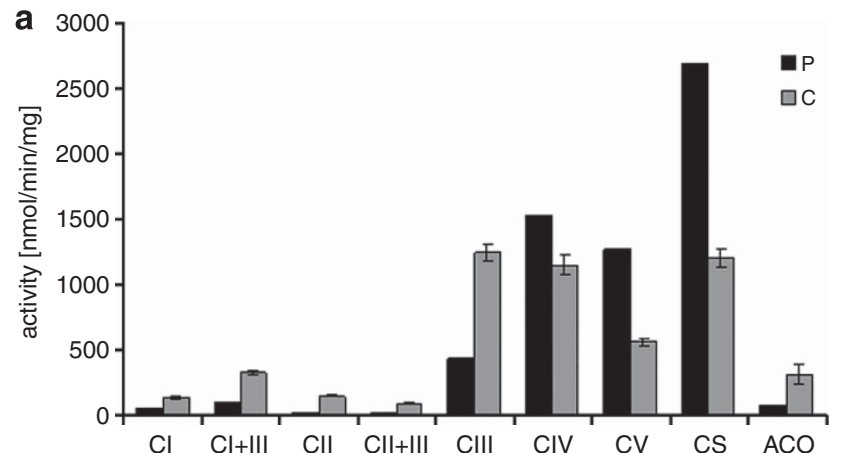

b

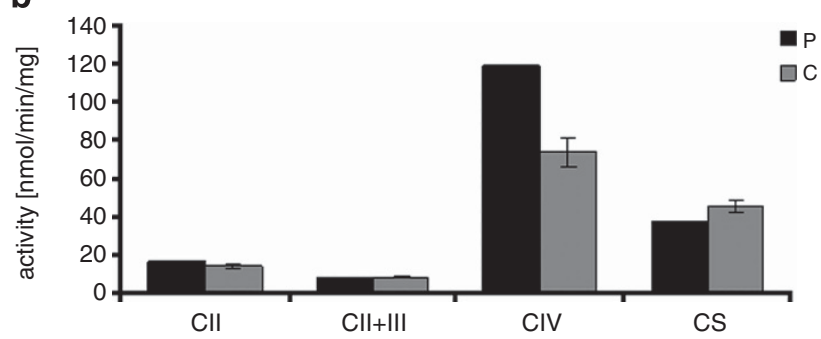

Figure 2 Enzymatic activities in muscle mitochondria and fibroblast homogenates. Respiratory chain complexes I-V (C I-IV), citrate synthase (CS) and aconitase (ACO) from control (C) and patient (P) were measured in isolated muscle mitochondria (a), whereas respiratory chain complexes II, $\mathrm{II}+\mathrm{III}$ and citrate synthase were measured in fibroblast homogenate (b), by spectrophotometric methods. The graphs depict mean \pm SEM of controls $(n>5)$.

synthase activity was above normal (Figure 2a). In fibroblasts homogenate, complex II and II + III respiratory chain activities when normalized to complex IV activity were moderately affected (69\% and $61 \%$, respectively; Figure $2 \mathrm{~b}$ ). PDHc activity in muscle homogenate was decreased to $0.9 \mathrm{nmol} / \mathrm{min} / \mathrm{mg}$ (control range $2.6-9.2 \mathrm{nmol} / \mathrm{min} /$ $\mathrm{mg}$ ), whereas lipoamide dehydrogenase (subunit E3 of PDHc) activity was within the normal range $118 \mathrm{nmol} / \mathrm{h} / \mathrm{mg}$ (control $99-237 \mathrm{nmol} /$ $\mathrm{min} / \mathrm{mg}$ ).

\section{Exome sequencing}

Exome analysis consisted of 38.6 million reads, which were confidently aligned to the human reference assembly HG19. Variant calling resulted in 55618 SNVs and 2856 small Indels. Of these, 8576 SNVs were non-synonymous variants but only 579 were not present in dbSNP135.

As the patient was the product of consanguineous marriages, we searched for a founder mutation and filtered out all heterozygous changes. Thirty-seven SNVs and five Indels survived but only two of them resided in genes encoding mitochondrial proteins - p.T175A in the MCU gene and chr19: 10426672 c.1 A>T, (p.0?) in the FDX1L gene (GI:72534754). Sanger sequencing of the two mutations in the family members excluded the MCU mutation, which did not segregate with the disease in the family and confirmed the FDX1L mutation (Figure 1b). We focused on this mutation, although it was predicted to be a benign polymorphism by the prediction software Mutation Taster ${ }^{5}$ and 'possibly damaging' by Polyphen, ${ }^{6}$ because the mutation affected a conserved residue and was not present in the 6503 exome data deposited at the Exome Variant Server, NHLBI GO Exome Sequencing Project (ESP), Seattle, WA (URL: http:// evs.gs.washington.edu/EVS/; Version: v.0.0.17. (November 30, 2012) a
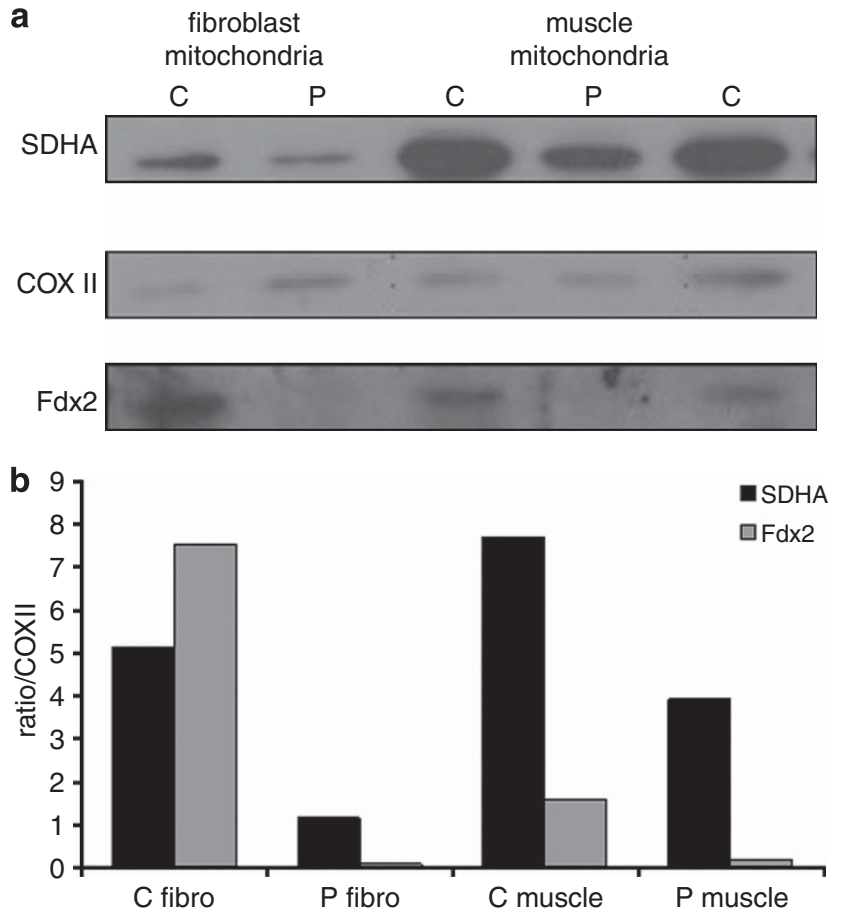

Figure 3 Immunoblot analysis. Isolated mitochondria from control (C) and patient (P) fibroblast and muscle (15 and $20 \mu \mathrm{g}$ protein, respectively) were subjected to SDS-polyacrylamide gel electrophoresis, processed for immunoblotting and probed with antibodies against SDHA, COXII and Fdx2 and detected by ECL (a). The amount of SDHA and Fdx2 proteins in the patient was estimated relatively to COXII and compared with controls (C muscle - depicts the mean value of two controls) (b).

accessed 01 2013). Using Sanger sequencing, we detected one carrier among 144 anonymous individuals of the same ethnic origin.

\section{Immunoblotting}

Immunoblot analysis showed an almost undetectable amount of the Fdx2 protein in the patients' fibroblast and muscle mitochondria (Figure 3a). Notably, the amount of complex II, probed by antibodies against SDHA, was decreased to $22-50 \%$, normalized to complex IV and compared with controls (Figure $3 \mathrm{~b}$ ).

\section{DISCUSSION}

Our patient presented with an adolescent onset proximal muscle myopathy associated with recurrent episodes of myoglobinuria and lactic acidosis consistent with mitochondrial myopathy. Biochemical assessment of her skeletal muscle confirmed severely deficient activities of complex I, II and III respiratory chain complexes with sparing of complexes IV and V. The fact that aconitase activity (a FeS-containing Krebs cycle enzyme) was also affected, whereas citrate synthase (a Fe-S independent Krebs cycle enzyme) was elevated, affirmed the suspicion of a mitochondrial Fe-S-related defect. This is also in accord with the finding of decreased PDHc as its E2 subunit is dependent on the Fe-S-dependent lipoate synthase (the E3 subunit is also assayed separately in the presence of external lipoic acid and is therefore normal). These clinical and biochemical phenotype highly resembles the previously reported myopathy caused by mutations in the ISCU gene encoding the Fe-S cluster scaffold protein ${ }^{8}$ (also known as myopathy with deficiency of SDH and aconitase). We therefore carried on with sequence analysis of this gene including the coding exons and their splice site consensus sequences and identified no 
mutation. Nevertheless, in keeping with the previously reported deep intronic mutation in this gene, ${ }^{8}$ which could have been missed with the above sequencing strategy and given the known consanguinity in the family, we employed a genome-wide homozygosity mapping, which eliminated autozygosity in the ISCU gene region. At this stage, we elected to proceed with exome sequencing, which identified the homozygous base pair substitution $\mathrm{c} .1 \mathrm{~A}>\mathrm{T}$ predicted to disrupt the ATG translation initiation site of the FDX1L gene.

More than 400 unique single base-pair substitutions, located within the ATG translation initiation codons of human genes, have been so far reported to cause genetic disease in humans. ${ }^{9}$ The process of protein translation initiates in almost all human mRNA at AUG codons (corresponding to ATG at the DNA level) resulting in methionine, the primary amino acid in most cellular produced proteins. ${ }^{10}$ The initiation site is reached via a ribosome-mediated scanning mechanism. Thus, the ribosome scans in a $5^{\prime}$ to $3^{\prime}$ direction until it reaches the AUG nearest to the $5^{\prime}$ end of the mRNA. ${ }^{11}$ This AUG is adopted as the initiation site but is only recognized efficiently when it is embedded within a suitable recognition sequence, also known as the 'Kozak consensus sequence' the optimal form of which approximates to $5^{\prime}$-GCCRCCatgG-3' (R: A or G), where the most frequently conserved bases in human mRNAs are a purine (R) at position -3 and a $G$ at position $+4 .{ }^{11}$ The mutation identified in our patient c.1A $>$ T causes the loss of the primary start codon ATG for Met1, which is replaced by a then un-translated triplet TTG coding for Leu. When a mutation alters the start codon, the scanning mechanism should activate initiation from the next downstream ATG. ${ }^{12}$ The next putative in frame start codon is present only 14 codons downstream coding for Met5, however, this methionine residue completely lacks the surrounding Kozak sequence, making it very unlikely alternative initiation site. In agreement with the predicted deleterious effect of the mutation on the translation of the protein, immunoblot assay of the patients' muscle and fibroblasts demonstrated lack of $\mathrm{Fdx} 2$ band as compared with controls (Figure 3a). Although quite unlikely, we cannot exclude that a shortened protein is produced but is rapidly degraded, and not detectable by immunoblot blot analysis.

FDX1L gene encodes a 183 amino acids mitochondrial protein, $\mathrm{Fdx} 2$, one of two mammalian $2 \mathrm{Fe}-2 \mathrm{~S}$ ferredoxins involved in $\mathrm{Fe}-\mathrm{S}$ cluster biogenesis. ${ }^{13}$ Fe-S clusters are evolutionary conserved, ubiquitously expressed, cofactors which are composed of iron and sulfur. They usually ligate to prosthetic proteins by the sulfhydryl side chains of their cysteine and often function as electron acceptors or donors playing a major role in crucial cellular activities. These include mitochondrial respiratory chain, which contains 12 Fe-S clusters in respiratory complexes I-III, and heme biosynthesis. In addition, Fe-S clusters are important in the proper enzymatic function of both the cytosolic and mitochondrial form of aconitase. At least three instances of autosomal recessive disruption in genes playing an essential role in $\mathrm{Fe}-\mathrm{S}$ biogenesis result in human phenotype accompanied by characteristic defects in complexes I, II and III of the mitochondrial respiratory chain. ${ }^{14,15}$ In agreement with this, targeted disruption of fdx2 and NFU1, another late-acting maturation factor for a subset of mitochondrial $\mathrm{Fe}-\mathrm{S}$ proteins, resulted in severely decreased content and activity of mitochondrial complex II (SDH) and the mitochondrial and cytosolic aconitases (only in fdx2 depletion) as well as impairment of lipoic acid containing enzymatic complexes. ${ }^{16,17}$ Indeed, we also detected similar deficiencies in our Fdx2-deficient patient indicating that both SDH functional (enzyme activity) and content (immunoblotting) are affected (Figure 2). Although SDHA was decreased in our patient, SDHB may even be more affected, being the Fe-S containing subunit, as it was previously demonstrated in ISCU myopathy patients. ${ }^{18}$ The decreased enzyme activities of complexes I and III, aconitase and PDHc in muscle biopsy are also consistent with Fe-S defect.

Notably, the mutation identified in our patient was predicted benign variant by the Mutation Taster software. This program, although scored highest for its accuracy, still holds $\sim 10 \%$ false prediction. ${ }^{5}$ Identification of DNA sequence alteration as disease causing is an intricate process. It combines appropriate segregation in the specific family, complete understanding of the consequences at the protein level, and in vivo and/or in vitro demonstration of pathogenicity of this variant. Appropriate recognition as 'disease causing' by a Mutation Prediction program is an additional support in the complex process of mutation validation. As exemplified here, prediction software may misdiagnose true mutations and therefore one should not rely exclusively on their results.

Finally, the phenotype displayed by our patient significantly resembles the ISCU myopathy both in its clinical and biochemical features. ${ }^{14}$ One difference was the muscle biopsy EM findings, which were normal in our patient and typically show dense mitochondrial inclusions in ISCU myopathy. ${ }^{18}$ This discrepancy may be related to the occasion the biopsy was taken when the patient was recovering and virtually asymptomatic. IscU is the scaffold protein on which the $\mathrm{Fe}-\mathrm{S}$ cluster is built, and as previously shown in an in-vitro system, this process is facilitated by ferredoxins. ${ }^{19}$ The isolated and relatively mild skeletal muscle phenotype in ISCU myopathy was elegantly explained by tissue-specific splicing of IscU. ${ }^{20} \mathrm{Fdx} 2$ is ubiquitously expressed and therefore the isolated skeletal muscle phenotype is perplexing. It may suggest that $\mathrm{Fdx} 2$ is not a vital component in $\mathrm{Fe}-\mathrm{S}$ biogenesis. A possible explanation may rely on the second ferredoxin Fdx1, which was recently demonstrated like Fdx2, to be important for Fe-S biogenesis. ${ }^{13}$ We speculate that either Fdx1 or another, yet unidentified factor, may compensate for Fdx2 deficiency, but when exposed to extreme conditions such as physical exercise it may be exhausted leading to rhabdomyolysis. Then again, the initiation site at Met 14, although probably inefficient may serve as a partial alternative leading to a modified predominantly skeletal muscle phenotype. Further studies in the future including studies that will deeply investigate tissue specificity are needed to resolve this issue.

Taken together, we suggest adding genetic analysis of FDX1L to the diagnostic work-up of patients with mitochondrial myopathy in general and specifically in patients displaying myopathy with deficiency of SDH and aconitase.

\section{CONFLICT OF INTEREST}

The authors declare no conflict of interest.

\section{ACKNOWLEDGEMENTS}

We thank Professor Roland Lill for generously donating anti-Fdx2 antibodies. This work was in part financed by the Israeli Ministry of Health Grant \#5914 and by the Manackerman Charitable Trust Fund UK. Sarah Weissman and Corinne Belaich are acknowledged for excellent technical assistance.

\footnotetext{
1 Saada A, Bar-Meir M, Belaiche C, Miller C, Elpeleg O: Evaluation of enzymatic assays and compounds affecting ATP production in mitochondrial respiratory chain complex I deficiency. Anal Biochem 2004; 335: 66-72.

2 Reisch AS, Elpeleg O: Biochemical assays for mitochondrial activity: assays of TCA cycle enzymes and PDHc. Methods Cell Biol 2007; 80: 199-222.

3 Wang K, Li M, Hakonarson H: ANNOVAR: Functional annotation of genetic variants
} from next-generation sequencing data. Nucleic Acids Res 2010; 38: e164. 
4 Sherry ST, Ward MH, Kholodov M et al: dbSNP: the NCBI database of genetic variation. Nucleic Acids Res 2001; 29: 308-311.

5 Schwarz JM, Rodelsperger C, Schuelke M, Seelow D: MutationTaster evaluates disease-causing potential of sequence alterations. Nat Methods 2010; 7: 575-576.

6 Adzhubei IA, Schmidt S, Peshkin L et al: A method and server for predicting damaging missense mutations. Nat Methods 2010; 7: 248-249.

7 Suomalainen A, Elo JM, Pietiläinen KH et al: FGF-21 as a biomarker for musclemanifesting mitochondrial respiratory chain deficiencies: a diagnostic study. Lancet Neurol 2011; 10: 806-818.

8 Mochel F, Knight MA, Tong WH et al: Splice mutation in the iron-sulfur cluster scaffold protein ISCU causes myopathy with exercise intolerance. Am J Hum Genet 2008; 82: 652-560.

9 Wolf A, Caliebe A, Thomas NS et al: Single base-pair substitutions at the translation initiation sites of human genes as a cause of inherited disease. Hum Mutat 2011; 32: 1137-1143.

10 Jackson RJ, Hellen CU, Pestova TV: The mechanism of eukaryotic translation initiation and principles of its regulation. Nat Rev Mol Cell Biol 2010; 11: $113-127$.

11 Lorsch JR, Dever TE: Molecular view of $43 \mathrm{~S}$ complex formation and start site selection in eukaryotic translation initiation. J Biol Chem 2010; 285: 21203-21207.

12 Kozak M: Pushing the limits of the scanning mechanism for initiation of translation. Gene 2002; 299: 1-34
13 Shi Y, Ghosh M, Kovtunovych G, Crooks DR, Rouault TA: Both human ferredoxins 1 and 2 and ferredoxin reductase are important for iron-sulfur cluster biogenesis. Biochim Biophys Acta 2012; 1823: 484-492.

14 Kollberg G, Tulinius M, Melberg A et al: Clinical manifestation and a new ISCU mutation in iron-sulphur cluster deficiency myopathy. Brain 2009; 132: 2170-2179.

15 Cameron JM, Janer A, Levandovskiy $V$ et al: Mutations in iron-sulfur cluster scaffold genes NFU1 and BOLA3 cause a fatal deficiency of multiple respiratory chain and 2-oxoacid dehydrogenase enzymes. Am J Hum Genet 2011; 89: 486-495.

16 Navarro-Sastre A, Tort F, Stehling 0 et al: A fatal mitochondrial disease is associated with defective NFU1 function in the maturation of a subset of mitochondrial Fe-S proteins. Am J Hum Genet 2011; 89: 656-667.

17 Sheftel AD, Stehling O, Pierik AJ et al: Humans possess two mitochondrial ferredoxins $\mathrm{Fdx} 1$ and Fdx2, with distinct roles in steroidogenesis, heme, and Fe/S cluster biosynthesis. Proc Natl Acad Sci USA 2010; 107: 11775-11780.

18 Haller RG, Henriksson KG, Jorfeldt $L$ et al: Deficiency of skeletal muscle succinate dehydrogenase and aconitase. Pathophysiology of exercise in a novel human muscle oxidative defect. J Clin Invest 1991; 88: 1197-1206.

19 Chandramouli K, Unciuleac MC, Naik S, Dean DR, Huynh BH, Johnson MK: Formation and properties of [4Fe-4S] clusters on the IscU scaffold protein. Biochemistry 2007; 46: 6804-6811.

20 Nordin A, Larsson E, Thornell LE, Holmberg M: Tissue-specific splicing of ISCU results in a skeletal muscle phenotype in myopathy with lactic acidosis, while complete loss of ISCU results in early embryonic death in mice. Hum Genet 2011; 129: 371-378. 